\title{
Ground State Structure of D75N Mutant of Sensory Rhodopsin II in Complex with its Cognate Transducer
}

\author{
A. Ishchenko ${ }^{\text {df }}$, E. Round ${ }^{\text {abce }}$, V. Borshchevskiy ${ }^{\text {abcde }}$, S. Grudinin ${ }^{\text {gh }}$, \\ I. Gushchin abce, J.P. Klarej, T. Balandin ${ }^{\mathrm{d}}$, A. Remeeva ${ }^{\mathrm{d}}$, M. Engelhard ${ }^{\mathrm{i}}$, G. Büldt ${ }^{\mathrm{de}}$, and V. \\ Gordeliy abcde*
}

${ }^{a}$ CEA, DSV, Institut de Biologie Structurale (IBS), Grenoble, F-38027, France.

${ }^{\mathrm{b}}$ CNRS, UMR 5075, 41 rue Jules Horowitz, Grenoble F-38027, France.

c Université Joseph Fourier, Grenoble 1, F-38000, France.

d Institute of Complex Systems (ICS), ICS-5: Molecular Biophysics, Research Centre Juelich, 52425 Juelich, Germany

e Research-educational Centre "Bionanophysics", Moscow Institute of Physics and Technology, 141700 Dolgoprudniy, Russia

${ }_{\mathrm{f}}^{\mathrm{f}}$ Institute of Crystallography, University of Aachen (RWTH), Jaegerstrasse 17-19, 52056 Aachen, Germany g NANO-D, INRIA Grenoble-Rhone-Alpes Research Center, 38334 Saint Ismier Cedex, Montbonnot, France

${ }^{\text {h }}$ CNRS, Laboratoire Jean Kuntzmann, BP 53, Grenoble Cedex 9, France

${ }^{i}$ Max-Planck Institute of Molecular Physiology, 44227 Dortmund, Germany

j Department of Physics, University of Osnabrück, Barbarastrasse 7, D-49069 Osnabrück, Germany

* Author for correspondence: valentin.gordeliy@ibs.fr

Tel: +33 438 780644, Fax: +33 438785494

41 rue Jules Horowitz, 38027 Grenoble Cedex 1 - France 


\begin{abstract}
The complex of sensory rhodopsin II (NpSRII) with its cognate transducer (NpHtrII) mediates negative phototaxis in halobacteria N. pharaonis. Upon light activation NpSRII triggers, by means of NpHtrII, a signal transduction chain homologous to the two component system in eubacterial chemotaxis. Here we report on the crystal structure of the ground state of the mutant NpSRII-D75N/NpHtrII complex in the space group I2 $22_{12} 2_{1}$. Mutations of this aspartic acid in lightdriven proton pumps dramatically modify or/and inhibit protein functions. However, in vivo studies show that the similar D75N mutation retains functionality of the NpSRII/NpHtrII complex. The structure provides the molecular basis for the explanation of the unexpected observation that the wild and the mutant complexes display identical physiological response on light excitation.
\end{abstract}

\title{
Keywords
}

Membrane protein; signal transduction; retinal protein; X-ray crystallography; protein crystallization

\section{Abbreviations}

SRII, sensory rhodopsin II; HtrII, cognate transducer protein of sensory rhodopsin II; Np, Natronomonas pharaonis; Hs, Halobacterium salinarum 


\section{Introduction}

Microbial rhodopsins are seven alpha-helical transmembrane proteins [1,2]. Although structurally highly similar, they are functionally quite diverse as they can act either as ion pumps, cation channels, or sensors $[3,4]$. They often serve as model systems to study ion transport or signal transfer across membranes. The recent interest to microbial rhodopsins is also related to a new field of optogenetics which has been proven especially valuable in neurophysiological research [5] and where rhodopsins play an essential role.

A very well studied model system for signal transfer across membrane is sensory rhodopsin II (NpSRII) in complex with its cognate transducer (NpHtrII), which mediates negative phototaxis in archaebacteria $N$. pharaonis. This complex displays a 2:2 stoichiometry where two transducers are flanked by two receptors. Upon light activation NpSRII triggers by means of NpHtrII a signal transduction chain homologous to the two component system in eubacterial chemotaxis [6]. The events following light activation have been studied quite extensively [7]. The absorption of a photon by the all-trans retinal chromophore, which is bound to the protein via a protonated Schiff base, leads to an all-trans to 13-cis isomerization. This initial event in turn triggers deprotonation of the Schiff base and, concomitantly, protonation of the Schiff base counter ion - Asp75. This sequence of events has also been observed for the ion pumps like bacteriorhodopsin [8]. Further step in the chain of signal transfer is the helix F movement [9] which leads to the rotation of the transducer transmembrane helix 2 (TM2) $[10,11]$. It was shown that the proton transfer from the Schiff base to Asp75 is not a mandatory step in the signal transduction from receptor to transducer. N. pharaonis carrying the mutation D75N in NpSRII, which inhibits the deprotonation of the Schiff base, still shows wild type behavior $[12,13]$ and it has been shown that NpSRII-D75N mutation preserves the movement of helix F [14] during the photocycle. Additionally, FTIR experiments 
showed that photocycle related conformational changes in wild type and D75N mutant are quite similar indicating that the neutralization of the Schiff base counter ion does not interfere with the formation of the active state [15]. Here we present the crystal structure of the ground state of the mutant NpSRII-D75N/NpHtrII complex in the space group $I 2{ }_{1} 2{ }_{1}{ }_{1}$ that provides us with additional information about signal transduction.

\section{Material and methods}

The genes of N. pharaonis SRII and HtrII truncated at C-terminus (1-157) were cloned into the pET27bmod expression vector with a C-terminal $\mathrm{x} 7$ His tag. The D75N mutant was prepared by PCR using the overlap-extension method [16]. E. coli cells were transformed by electroporation [17].

Proteins were expressed in Escherichia coli strain BL21 (DE3), and purified as described [18]. After removal of imidazole by diethyl-aminoethyl chromatography, SRII-D75N and HtrII157 were mixed in a 1:1 ratio, followed by the reconstitution into purple membrane (the bacteriorhodopsin containing membrane patches of $\mathrm{H}$. salinarum) lipids (protein to lipid ratio 1:35). After filtration, the reconstituted proteins were pelleted by centrifugation at $100,000 \mathrm{~g}$. For resolubilization, the samples were resuspended in a buffer containing $150 \mathrm{mM} \mathrm{NaCl}, 25 \mathrm{mM} \mathrm{Na} / \mathrm{K}$ phosphate $\mathrm{pH} 5.1,2 \%$ n-octyl-b-D -glucopyranoside and shaken for $16 \mathrm{~h}$ at $4^{\circ} \mathrm{C}$ in the dark. The rest of lipids were pelleted by centrifugation at 100,000g while the solubilized SRII/HtrII157 complex stayed in the solution.

The solubilized protein complex was added to the lipids in crystallization buffer $(150 \mathrm{mM}$ $\mathrm{NaCl}, 25 \mathrm{mM} \mathrm{Na} / \mathrm{K}$ phosphate $\mathrm{pH} 5.1,0.8 \%$ n-octyl-b-D -glucopyranoside). The liposomes with the complex were mixed with monovaccenin or monooleoyl (Nu-Chek Prep) to form mesophase 
[19]. Best crystals were grown with the precipitant $1 \mathrm{M} \mathrm{Na} / \mathrm{K}$ phosphate buffer, pH 5.6. Crystallization trials were kept at $22^{\circ} \mathrm{C}$.

X-ray diffraction data were collected at beamline ID14-1 of the European Synchrotron Radiation Facility (ESRF), Grenoble, France, using a Quantum ADSC Q4R CCD detector. Data were integrated using MOSFILM [20] and scaled with SCALA [21] from the CCP4 program suite [22]. Molecular replacement was performed using MOLREP [23] for a polyalanine model (from PDB accession number $1 \mathrm{H} 2 \mathrm{~S}$ ) and gave a unique solution. Starting from a polyalanine model of NpSRII/NpHtrII (Protein Data Bank accession code 1H2S) the molecular replacement solution was completed by the automated refinement procedure (ARP/wARP [24]). Residues 2-221 of SRIID75N and residues 23-84 of HtrII were built.

\section{Results}

We have obtained the NpSRII-D75N/NpHtrII mutant structure from crystals diffracting to $\sim 2 \AA$ grown by a in meso approach. The crystals were plate-like and grew up to $1200 \times 50 \times 20 \mu \mathrm{m}$ (see SI for crystallographic statistics). Protein molecules are arranged in parallel layers with protein crystal belonging to type I [25]. Each asymmetric unit contains one NpSRII-D75N/NpHtrII heterodimer, one of the crystallographic axes is collinear to the natural 2-fold axis between transducer molecules in the 2:2 dimer. The complex is "U"-shaped as the two NpSRII-D75N/ NpHtrII heterodimers are parallel to each other. It is in contrast to the "V"-shape of our previous structure of this complex [26]. We suppose that the "U"-shape enables a better interaction interface between the transducer and the receptor than the "V"-shape (refer to "Ishchenko et al. New Insights on Signal Propagation by Sensory Rhodopsin II/Transducer Complex, to be published (2013)" for further details). 
The mutation was done in the extracellular part of the protein and we observed no significant structural differences between cytoplasmic parts of D75N mutant and wild type. Detailed comparison of active sites of NpSRII-D75N/NpHtrII and wild type ground, K- and Mstates are shown in Fig.2. Several conformational features of NpSRII-D75N/NpHtrII resemble conformational changes in the M-state of the wild type. In comparison with wild type Asp75, Asn75 of the NpSRII-D75N/NpHtrII does not form hydrogen bonds with water molecule W1 and the Schiff base. As a consequence we observe the rotation of Asn75 side chain with respect to Asp75 and its shift towards Arg72. The mutated amino acid loses its hydrogen bond to Thr79. Water molecule W3 disappears breaking more bonds in the hydrogen bond network (see Table 1). Water molecule W2' is moved by $0.9 \AA$ towards the Schiff base. It forms a hydrogen bond with the nitrogen atom of Asn75 resembling W2'-Asp75 H-bond in the wild type M-state. Water molecule W4' is shifted in the mutant in extracellular direction by $2 \AA$. Thereby the water-mediated link between Arg72 and Tyr51 is broken. There is a slight movement of Arg72 residue towards the extracellular part which can be related to the absence of the negative charge at Asn75. Water molecule W1 is $0.3 \AA$ shifted towards the Schiff base. All these rearrangements lead to the G helix bending at residues 196-206 with the maximum amplitude of $0.3 \AA$ around Asn201.

In the ground state of the wild type water molecule W3 together with W1, Asp75, Asp201 and the Schiff base forms a pentagon of hydrogen bonds. Disappearance of W3 in the NpSRIID75N/NpHtrII destroys the pentagon structure and weakens the connection between $\mathrm{C}$ and $\mathrm{G}$ helices. Similar weakening of the C-G-helix interaction is observed in the transition of the wild type complex to K- and M-states where W3 is destabilized along with W1. Reduced C-G interaction in the mutant is not sufficient to trigger the rearrangement as in the active states of wild type. The hydrogen bond network stabilizing the ground state structure of mutant is loosened and is somewhat 
shifted towards signaling states of the wild type. Nevertheless, it is strong enough to keep the position of helices. Additional energy from photoisomerization of the retinal is necessary to trigger the signal transduction process.

\section{Discussion}

Basing on the assumption that the signaling states of NpSRII-D75N/NpHtrII are formed on the same basis as for the ground state complex we can foresee how the signal is generated in the mutant complex structure. According to the current understanding of the signal transduction mechanism [11] retinal isomerizes upon absorption of a photon and the hydrogen bond between Schiff base and the adjacent water molecule W1 is broken resulting in its disappearance from the active site. Side chains of the residues in the active site become mobile as can be seen from increased B-factors in active states [11,27]. The carboxylic group of Asp75 is rotated by $90^{\circ}$ with respect to its ground-state position thereby losing its connection to the water molecule W3, which becomes disordered. Such reduction of the hydrogen bond network in the extracellular part of retinal pocket leads to further structural rearrangements. The water molecule W2 shifts towards Asp75 in the M-state of wild type NpSRII/NpHtrII complex. In the ground state it is linked to the water molecules W3 and W4 and to O $\delta$ of Asp201. The new position of Asp75 allows it to create Hbond with W2. Thus, only two hydrogen bonds are mediated by W2 in the M-state in contrast to three bonds in the ground state. Water molecule W4 shifts in the M-state in the extracellular direction by $2 \AA$ concomitantly breaking water-mediated link between Tyr51 and Arg72 (see Table 1). Finally, interhelical connections between helices $C$ and G are broken and the receptor splits in two loosely connected domains consisting of A, B and G $\alpha$-helices and C, D, E and F, respectively. The movement of the helix G triggers signal propagation to the TM2 helix of the transducer. It should be noted, that according to ssNMR data the overall structure of wild type NpSRII and its 
D75N mutant are quite similar. Changes observed are consistent with a rearrangement of the hydrogen bonding network close to retinal Schiff base [28].

Data presented here allows us to deduce a molecular mechanism of signal transfer from the receptor to the transducer in NpSRII-D75N/NpHtrII. It was discussed above that the NpSRII D75N mutant is different from the wild type protein mainly in the hydrogen bond network, which in fact weakens the $\mathrm{C}$ and G-helices interaction. Similar weakening is also observed during the transition to the K-intermediate of the wild type [11]. In both instances the proteins are capable for transition to the signaling state. Infrared spectroscopic data indicated that the conformational changes observed upon activation of the NpSRII-D75N mutant are almost identical to those observed for the wild type [15]. It is in line with the observation that this mutant displays wild type physiological behavior and gives additional confidence for the structural explanation of the unexpected consequence of D75N mutation described above. From electron paramagnetic resonance data it was concluded that the D75N mutation shifts the ground state conformation of NpSRII-D75N and its cognate transducer into the direction of the signaling state [14].

The present results and those from spectroscopic investigations could provide a reason why the corresponding HsSRII-D73N mutant shows constitutive activity. In this case, neutralization of the counterion might trigger conformational changes large enough to destabilize the ground state. This leads to the spontaneous activation of the mutant complex without triggering by light that is manifested in 3-fold motion reversals activity in H. salinarum cells [12]. In the case of HsSRII the mutation of the counterion reduces the energy of the transition from ground to the active state to that comparable to the energy of thermal fluctuations. On the contrary, changes in the structure of NpSRII-D75N are quite small comparing to the wild type and the ground state structure is stabilized by the hydrogen bonds. 
Summarizing, the crystal structure of the ground state complex of NpSRII-D75N/NpHtrII in the crystals of the $I 2{ }_{1} 2_{1} 2_{1}$ space group provides a new insight into the molecular mechanisms of signal propagation from NpSRII to its cognate transducer NpHtrII. We show the importance of the hydrogen bonds network inside SRII and propose a mechanism by which the energy stored in hydrogen bonds is transferred to NpHtrII. It should be stressed that these data do not contradict with the early proposed molecular mechanism of signal development in the membrane part of the NpSRII/NpHtrII complex [11]. In opposite, the present work provides an additional valuable support.

\section{Accession numbers}

Coordinates and structure factors have been deposited in the Protein Data Bank with the 4GYC accession number. 


\section{Cytoplasmic side}

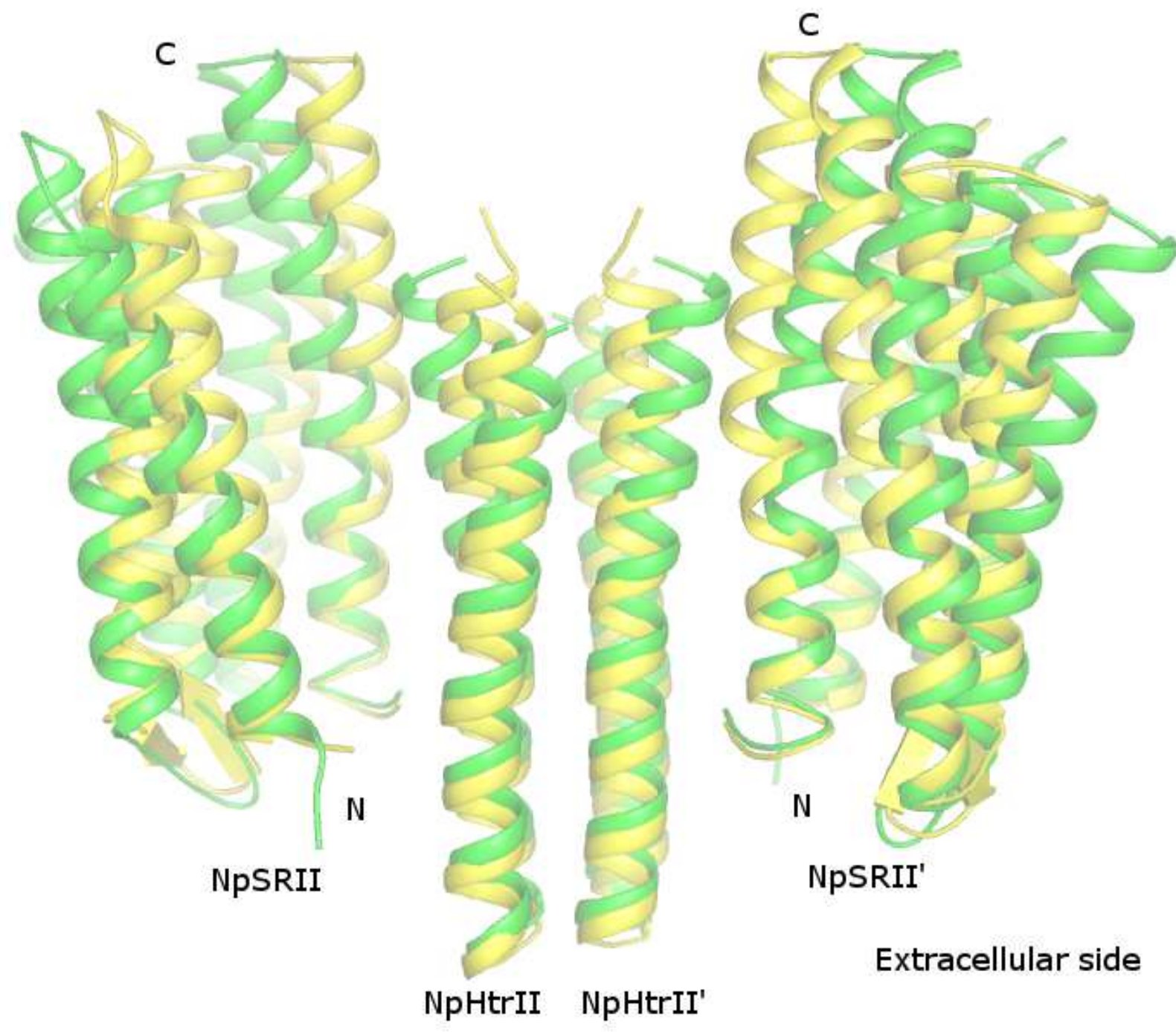

Figure 1

Fold of the receptor/transducer complex (side view). NpSRII-D75N/NpHtrII complex is shown in yellow and the wild type NpSRII/NpHtrII (PDB ID 1H2S) is shown is green. "N" and "C" letters denote $\mathrm{N}$ - and C-terminus of the proteins. Coordinates and structure factors of NpSRIID75N/NpHtrII have been deposited in the Protein Data Bank with the 4GYC accession number. 

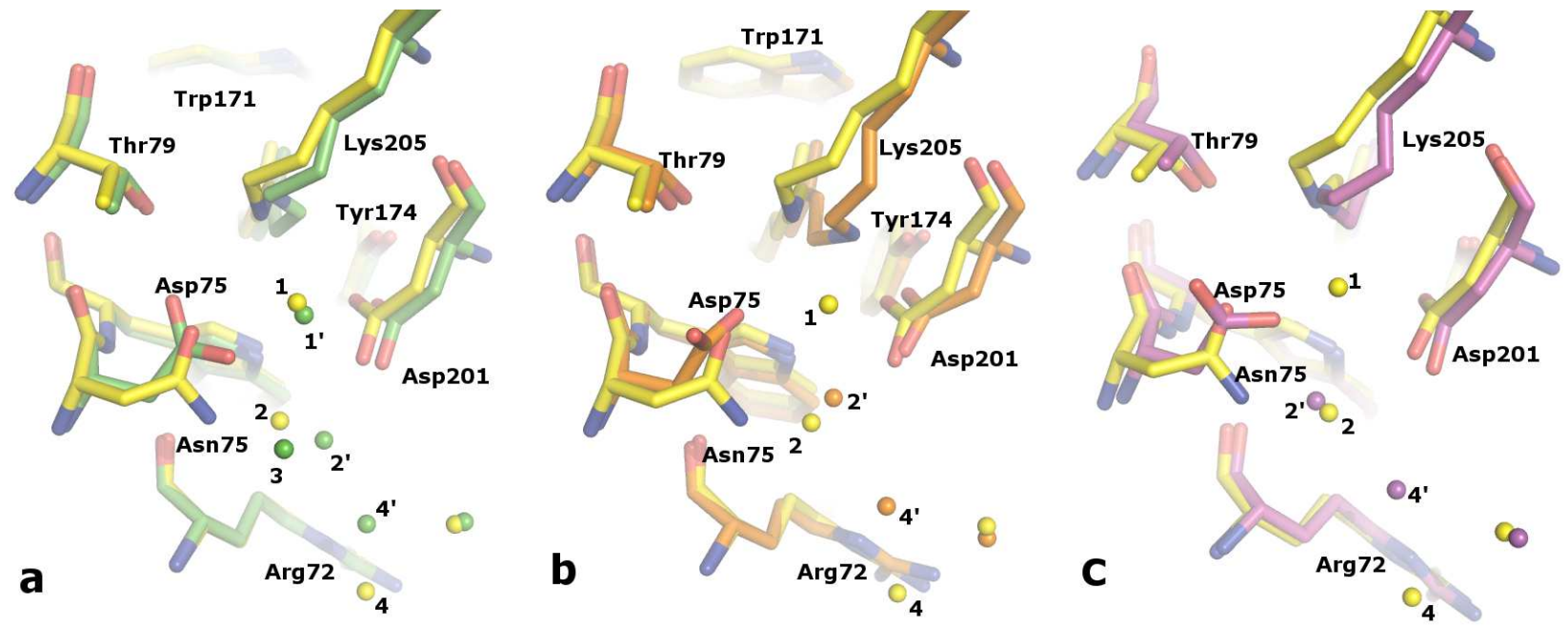

Figure 2

Panel a: Structural differences between the ground states of NpSRII-D75N and WT (PDB ID $1 \mathrm{H} 2 \mathrm{~S}$ ) in the vicinity of the retinal Schiff base including water molecules,. Wild type molecule and waters (balls) are shown in green; NpSRII-D75N is shown in yellow. Primes denote water molecules in the wild type ground structure.

Panel b: Structural differences between NpSRII-D75N ground state and the K-state intermediate (PDB ID 2F93) in the vicinity of the retinal Schiff base including water molecules. Ground state NpSRII-D75N and waters (balls) are shown in yellow, the K-state and waters are shown in orange. Primes denote water molecules in the K-state structure.

Panel c: Structural differences between NpSRII-D75N ground state and the M-state intermediate (PDB ID 2F95) in the vicinity of the retinal Schiff base including water molecules. Ground state NpSRII-D75N and waters (balls) are shown in yellow, the M-state and waters are shown in magenta. Primes denote water molecules in the M-state structure. 


\section{NpSRII/NpHtrII N NpSRII-D75N/NpHtrII}

ground state, PDB ID ground state, PDB ID

NpSRII/NpHtrII M-

state, PDB ID 2F95

\begin{tabular}{|c|c|c|c|}
\hline & $1 \mathrm{H} 2 \mathrm{~S}$ & $4 \mathrm{GYC}$ & \\
\hline W1・••Asp75/Asn75 & + & + & - \\
\hline W1・•Asp201 & + & + & - \\
\hline W1•••Lys205 & + & + & - \\
\hline W2•••Asp201 & + & + & + \\
\hline W2・••W3 & + & - & - \\
\hline W2・••W4 & + & - & - \\
\hline W2・••Asp75/Asn75 & - & + & + \\
\hline W3・••Asp75/Asn75 & + & - & - \\
\hline W4•••Tyr51 & + & - & - \\
\hline W4・•・Arg72 & + & + & + \\
\hline
\end{tabular}

Table 1. List of hydrogen bonds in the active center for different states of the complex.

\section{Acknowledgments}

We are thankful to E. Pebay-Peyroula for support of this project and C. Baeken for the assistance with protein preparation. We acknowledge Structural biology group of the European Synchrotron Radiation Facility for provision of synchrotron radiation facilities. The work was supported by the program "Chaires d'excellence" edition 2008 of ANR France, CEA(IBS) HGF(FZJ) STC 5.1 specific agreement, the MC grant (Marie Curie, FP7-PEOPLE-2007-1-1-ITN, project SBMPs) and an EC FP7 grant for the EDICT consortium (HEALTH-201924). Part of this work was supported by BMBF (PhoNa - Photonic Nanomaterials). The work was partially supported by the Federal Target Program «Scientific and academic research cadres of innovative Russia» for 2009-2013 years. We greatly acknowledge support of this work by ONEXIM, Russia. 


\section{References}

[1] A.K. Sharma, J.L. Spudich, W.F. Doolittle, Microbial rhodopsins: functional versatility and genetic mobility., Trends in Microbiology. 14 (2006) 463-9.

[2] T.M. Bridges, C.W. Lindsley, G-protein-coupled receptors: from classical modes of modulation to allosteric mechanisms., ACS Chemical Biology. 3 (2008) 530-41.

[3] W.D. Hoff, K.H. Jung, J.L. Spudich, Molecular mechanism of photosignaling by archaeal sensory rhodopsins., Annual Review of Biophysics and Biomolecular Structure. 26 (1997) $223-58$.

[4] D. Oesterhelt, The structure and mechanism of the family of retinal proteins from halophilic archaea., Current Opinion in Structural Biology. 8 (1998) 489-500.

[5] T. Knöpfel, M.Z. Lin, A. Levskaya, L. Tian, J.Y. Lin, E.S. Boyden, Toward the second generation of optogenetic tools., The Journal of Neuroscience : the Official Journal of the Society for Neuroscience. 30 (2010) 14998-5004.

[6] A.M. Stock, V.L. Robinson, P.N. Goudreau, Two-component signal transduction., Annual Review of Biochemistry. 69 (2000) 183-215.

[7] J.P. Klare, I. Chizhov, M. Engelhard, Microbial rhodopsins: scaffolds for ion pumps, channels, and sensors., Results and Problems in Cell Differentiation. 45 (2008) 73-122.

[8] J.K. Lanyi, Bacteriorhodopsin., Annual Review of Physiology. 66 (2004) 665-88.

[9] A.A. Wegener, I. Chizhov, M. Engelhard, H.J. Steinhoff, Time-resolved detection of transient movement of helix F in spin-labelled pharaonis sensory rhodopsin II., Journal of Molecular Biology. 301 (2000) 881-91.

[10] A.A. Wegener, J.P. Klare, M. Engelhard, H.J. Steinhoff, Structural insights into the early steps of receptor-transducer signal transfer in archaeal phototaxis., The EMBO Journal. 20 (2001) 5312-9.

[11] R. Moukhametzianov, J.P. Klare, R. Efremov, C. Baeken, A. Göppner, J. Labahn, et al., Development of the signal in sensory rhodopsin and its transfer to the cognate transducer., Nature. 440 (2006) 115-9.

[12] E.N. Spudich, W. Zhang, M. Alam, J.L. Spudich, Constitutive signaling by the phototaxis receptor sensory rhodopsin II from disruption of its protonated Schiff base-Asp-73 interhelical salt bridge., Proceedings of the National Academy of Sciences of the United States of America. 94 (1997) 4960-5.

[13] I. Chizhov, G. Schmies, R. Seidel, J.R. Sydor, B. Lüttenberg, M. Engelhard, The photophobic receptor from Natronobacterium pharaonis: temperature and $\mathrm{pH}$ dependencies of the photocycle of sensory rhodopsin II., Biophysical Journal. 75 (1998) 999-1009.

[14] J. Holterhues, E. Bordignon, D. Klose, C. Rickert, J.P. Klare, S. Martell, et al., The signal transfer from the receptor NpSRII to the transducer NpHtrII is not hampered by the D75N mutation., Biophysical Journal. 100 (2011) 2275-82. 
[15] M. Hein, I. Radu, J.P. Klare, M. Engelhard, F. Siebert, Consequences of counterion mutation in sensory rhodopsin II of Natronobacterium pharaonis for photoreaction and receptor activation: an FTIR study., Biochemistry. 43 (2004) 995-1002.

[16] R. Higuchi, B. Krummel, R.K. Saiki, A general method of in vitro preparation and specific mutagenesis of DNA fragments: study of protein and DNA interactions., Nucleic Acids Research. 16 (1988) 7351-67.

[17] W.J. Dower, J.F. Miller, C.W. Ragsdale, High efficiency transformation of E. coli by high voltage electroporation., Nucleic Acids Research. 16 (1988) 6127-45.

[18] I.P. Hohenfeld, A.A. Wegener, M. Engelhard, Purification of histidine tagged bacteriorhodopsin, pharaonis halorhodopsin and pharaonis sensory rhodopsin II functionally expressed in Escherichia coli., FEBS Letters. 442 (1999) 198-202.

[19] M. Caffrey, V. Cherezov, Crystallizing membrane proteins using lipidic mesophases., Nature Protocols. 4 (2009) 706-31.

[20] A.G.W. Leslie, The integration of macromolecular diffraction data., Acta Crystallographica. Section D, Biological Crystallography. 62 (2006) 48-57.

[21] P. Evans, Scaling and assessment of data quality., Acta Crystallographica. Section D, Biological Crystallography. 62 (2006) 72-82.

[22] N. 4 Collaborative Computational Project, The CCP4 suite: programs for protein crystallography., Acta Crystallographica. Section D, Biological Crystallography. 50 (1994) $760-3$.

[23] A. Vagin, A. Teplyakov, MOLREP : an Automated Program for Molecular Replacement, Journal of Applied Crystallography. 30 (1997) 1022-1025.

[24] G. Langer, S.X. Cohen, V.S. Lamzin, A. Perrakis, Automated macromolecular model building for X-ray crystallography using ARP/wARP version 7., Nature Protocols. 3 (2008) 1171-9.

[25] W. Avenue, N. Haven, Crystallization of membrane proteins Christian Ostermeier and Hartmut Michelt, (1995) 697-701.

[26] V.I. Gordeliy, J. Labahn, R. Moukhametzianov, R. Efremov, J. Granzin, R. Schlesinger, et al., Molecular basis of transmembrane signalling by sensory rhodopsin II-transducer complex., Nature. 419 (2002) 484-7.

[27] I. Gushchin, A. Reshetnyak, V. Borshchevskiy, A. Ishchenko, E. Round, S. Grudinin, et al., Active state of sensory rhodopsin II: structural determinants for signal transfer and proton pumping., Journal of Molecular Biology. 412 (2011) 591-600.

[28] M. Etzkorn, K. Seidel, L. Li, S. Martell, M. Geyer, M. Engelhard, et al., Complex formation and light activation in membrane-embedded sensory rhodopsin II as seen by solid-state NMR spectroscopy., Structure (London, England 300-293 (2010) 18 .).1993 :. 